\title{
Towards open access journals
}

Joan C. Bevan MD

\section{Canadian Journal of Anesthesia expanded elec- tronic archives}

The Canadian Journal of Anesthesia expanded electronic archives project was announced in January 2006. ${ }^{1}$ It had been developed over two years by our Editor-in-Chief, Donald Miller, after talks with HighWire Press about open-archiving policies being adopted by some biomedical journals. All articles published in the Journal can now be searched on the internet by authors' names, key words or publication dates (www.cja-jca.org). Citations are listed and there are links to full text and abstracts after January 1, 2000, with PDF files and abstracts for all articles from 1966 to 1999 . Articles are available only to subscribers, or on a pay-per-view basis to non-subscribers, for one year after publication. After that, they become freely available on the internet.

\section{Changing Journal policies}

This is not the first time that there has been a major change in the publication policies of the Journal. The "Proceedings of the Society", first published in 1952, became the quarterly "Canadian Anaesthetists' Society Journal" in 1954. This evolved into the "Canadian Journal of Anaesthesia" in 1987, and is now a monthly publication, the "Canadian Journal of Anesthesia". These name changes reflect an increasing readership and maturing editorial and business policies of the publication. The printed version has been complimented by the "The Electronic Anesthesia Library (TEAL )" CD ROM since 1997, and an online full-text format for the last seven years. The expanded electronic archives project is consistent with improving services to readers and respects the traditional business model of biomedical journals. It assumes that key subscription and advertising revenues are based upon protection of the most recent $6-12$ months of material. There will be no anticipated revenue loss, with only modest costs for optical scanning of PDF files and ongoing hosting charges.

\section{Emergence of electronic journal publication}

Traditional print-only publication of biomedical journals is being challenged by the emergence of electronic publication and internet distribution. ${ }^{2}$ The digital medium offers the alternative of faster dissemination of unlimited amounts of information, with the elimination of printing and distribution costs. It is seen as a solution to the "serials crisis", brought about by the rapid growth in the number of biomedical journals in the 1990s, higher production costs, publication delays, rising journal prices to individual subscribers, and institutional budget restrictions that limit the ability of libraries to purchase serials. Typically, smaller societies hosting subscription-based print journals have found it difficult to meet higher costs and some, such as "CRNA: The Clinical Forum for Nurse Anesthetists", ceased publication. ${ }^{3}$ As world-wide access to inexpensive internet technology becomes available, traditional publishing models are no longer viable.

Institutional repositories have been hosted on the internet to provide permanent archiving and allow easy access to out-of-print articles or electronic-only articles. Individual authors'articles or full journals can be posted on sites such as PubMed Central, which hosts over 200 journals including BMJ, CMAJ, Canadian Family Physician, and PloS Medicine (www.pubmedcentral.nih.gov). As more journals post articles and supplements online, few journals remain print-only. Between 2003 and 2005, publication of internet-only articles doubled from 7 tol4\% (60/861 to $128 / 917$ of all articles) in 138 high impact journals (including the "New England Journal of Medicine", "Pediatrics", "CMAJ" and "British Journal of Anaesthesia"). ${ }^{4}$ Most journals use hypertext to make electronic linkages to citations and send e-mail notifications to subscribers, but few have taken advantage of the potential for interactive functions. Nevertheless, reading habits of journal subscribers have changed: from dependence on printed journals and libraries to using computer access to literature from the internet.

CAN J ANESTH 2007 / 54: 7 / pp 501-508

From the Department of Anesthesia, University of Toronto, Toronto, Ontario, Canada.

Address correspondence to: Dr. Joan C. Bevan, Department of Anesthesia, University of Toronto, Room 126, FitzGerald Building, 15 College Street, Toronto, Ontario M5S 3E2, Canada. Phone: 416-978-4306; E-mail: joan.bevan@utoronto.ca 
Open access policies

The concept of open access (OA) publication has gained momentum over the last five years. ${ }^{5}$ This refers to the unrestricted distribution and availability of scholarly research and publications through the internet. Interested groups seek to promote OA: the Budapest Open Access Initiative (2002-2003), the Bethesda Statement on Open Access Publishing (2003) and the Berlin Declaration on Open Access to Knowledge in the Sciences and Humanities (2003). The groups subscribe to an OA policy for publication that goes beyond the simple free access to all full-text content in PubMed Central, to one that meets the following conditions: ${ }^{6}$

1) The author(s) and copyright holder(s) grant(s) to all users a free, irrevocable, worldwide, perpetual right of access to, and a license to copy, use, distribute, transmit and display the work publicly and to make and distribute derivative works, in any digital medium for any responsible purpose, subject to proper attribution of authorship, as well as the right to make small numbers of printed copies for their personal use.

2) A complete version of the work and all supplemental materials, including a copy of the permission as stated above, in a suitable standard electronic format is deposited immediately upon initial publication in at least one online repository that is supported by an academic institution, scholarly society, government agency, or other well-established organization that seeks to enable OA, unrestricted distribution, interoperability, and long-term archiving (for the biomedical sciences, PubMed Central is such a repository).

Definitions of OA may be debated, but any scholarly material that is OA should be freely accessible on the internet. In OA publishing, known as the "gold road", refereed journals may be archived in an institutional repository that makes their articles immediately available online. Open access self-archiving by authors, or the "green road", allows authors to make their articles freely accessible as post-prints or as non-peer reviewed pre-prints in addition to journal publication. ${ }^{2}$

\section{Copyright issues}

In print publication, proper attribution and use of published work is regulated by copyright laws. They protect the intellectual property of medical authors and aim to balance the rights of authors, publishers and copyright holders with society's right to knowledge. Copyright law allows "fair use" to copy, publish or distribute all or part of the work without the author's permission in certain circumstances. ${ }^{7}$ In Canada, authors of creative works, including software, automatically have the right to restrict access to oth- ers to use, publish, read and modify their work. There are no special provisions for electronic archives, but copyright laws are more difficult to enforce in the electronic medium. In OA publishing, authors may retain copyright for self-archived articles, but license agreements can override copyright. In the case of the Journal, the materials being archived were then, and remain now, under the copyright provisions of the Canadian Anesthesiologist's Society (CAS). In effect, the Editor-in-Chief, on behalf of the CAS, granted permission for HighWire to proceed with the project. The same copyright provisions apply to articles published in the Journal before 2000, as PDF files, or afterwards, in html format.

\section{Perceptions of OA publication}

Universal and free access to the scientific literature is perceived to be a public good that benefits mankind. While shared knowledge should promote research and education in developing countries, publishers argue that they already provide free journal content worldwide through international programs. Patient advocacy groups believe that they are entitled to see the results of publicly-funded research into specific diseases. Funding agencies have considered making grants conditional on $\mathrm{OA}$, but most major funding agencies in North America and the United Kingdom (National Institutes of Health, Social Sciences and Humanities Council, Canadian Institutes of Health Research, Genome Canada and Wellcome) who support OA, do not meet publication costs. For a time, NIH made deposition of publications in PubMed Central mandatory but, in 2005 , they changed this to a voluntary requirement after complaints from publishers. Initial compliance has been low, with only $2-3 \%$ of eligible publications posted on PubMedCentral. ${ }^{2}$

Timely world wide distribution of articles should increase visibility and readership, but the impact of $\mathrm{OA}$ on citation rates is not clear. One review of $\mathrm{OA}$ and non-OA articles published in the same journal, from June-December 2004, found that $14.2 \%$ were $\mathrm{OA}$ articles (with authors paying for publication) and were cited twice as often as non-OA articles. ${ }^{8}$ An electronic survey of authors who submitted to $B M J$, Archives of Diseases in Childhood, and the Journal of Medical Genetics in 2004 showed that authors were not very familiar with $\mathrm{OA}$ and this was not a consideration in their choice of journal for submission of papers. ${ }^{9}$ Authors want to have their work published in quality peer-reviewed journals, OA or not. As yet, there is no agreement among universities on whether OA publications should be used in tenure and promotion decisions. 


\section{Publishing costs}

Authors write manuscripts, using word processors, to produce electronic files that they send to editors via the internet. Editors distribute the manuscript to reviewers who return their comments, again via the internet. Modifications are made to the manuscript by the author, editor, copy editor and typesetter to produce the final electronic file, usually as a PDF. To this point the cost of preparing the final manuscript is the same for traditional print and electronic/internet journals. However, the cost of distribution of print journals is much more expensive than electronic/internet journals because of the additional costs of printing and mailing. The cost savings achieved in moving to internet-only publication by one indexed Canadian medical journal, Clinical and Investigative Medicine, allowed it to survive and continue publication.

Despite anticipated saving in printing and distribution costs, the move from print to electronic publication has not eliminated all publication costs. ${ }^{10}$ Proponents of print publication (or opponents of internet publication) argue that electronic publication does not reduce publication costs. It has been claimed that electronic publishing can be as expensive as the print medium, with the cost per article for both estimated at $\$ 3,000(\$ 1,000-6,000) .{ }^{11}$ The purchase price of journals is known, although favourable rates may be given for institutional subscriptions, bundled e-journals and site licences. ${ }^{12}$ Analysts have attempted to compare the publication costs of journals across disciplines, or between the for-profit and non-profit sectors, by calculating the median cost per article or citation based on pricing and the number of articles and citations listed in the International Standards Institute (ISI) Journal Citation Reports database (www.journalprices. com). Using 2002 ISI data and 2006 pricing (in US currency) for medical journals, the median cost per article or citation is three times greater in a for-profit journal than in a non-profit journal ( $\$ 3.1$ vs 10.5 per article or $\$ 2.9$ vs 8.5 per citation). The convenience of a price per article or citation calculation is attractive in exploring new business models to apply to internet publication. However, it reveals cost differences that cannot easily be explained.

Re-couping the costs of publication of scholarly journals used to depend on maintaining a subscriber base. Escalating journal prices have been blamed on higher publishing costs. However, the for-profit publishers maintain confidentiality of their budgets and profit margins that justify these increases. With OA publication, cost recovery often depends on an "author-pays (to publish)" or "user-pays (for view)" business model. The Public Library of Science charges authors or research sponsors a publication fee (waived if the author has no funds available) of \$1,250-2,500 per article to publish in their PLoS journals. This figure is based on expenses for manuscript processing and production, but not marketing, web-hosting or editorial staff costs. ${ }^{11}$ BioMed Central offers institutional annual memberships to cover these payments for their authors, but the pricing fluctuates according to the number of articles posted in the previous year. Nonprofit publications, such as society journals, operate at much lower budgets by reliance on volunteer peer reviewers and subsidized editorial office management. Advertising revenues in print journals represented profit that would support other activities for a society. These sources of funds have not yet been transferred into electronic publications. Profitability of the enterprise may have decreased accordingly, but this is not a change in the true production costs of the journal.

Editors can place little reliance on reported publication costs that vary so widely and must make business decisions that meet the needs of individual journals. Most established scholarly journals are still publishing print journals in addition to an internet version: thereby failing to maximize on potential cost reductions. New electronic-only journals have also proliferated; published by small groups or individual faculty on restricted budgets. These have been made possible by the free availability of journal management software systems, such as the federally funded Public Knowledge Project Open Journal Systems (OJS) pkp.sfu.ca/?q=ojs, that manage all aspects of a peerreviewed article.

\section{Predicting the future}

Making an analogy between scholarly electronic publishing and a fragile ecosystem in the environment, Forsman and Denison ${ }^{13}$ stated that. "When resources are widely available and ownership is unclear or unprotected, exploitation frequently occurs". Nevertheless, internet publication can serve the public need for access to information while retaining traditional values that ensure journal quality. If there is continuing pressure by granting agencies and government towards $\mathrm{OA}$, providing immediate public access to articles, clinical journals will risk loss of their subscription revenues. Cost efficiencies could result in publication of unedited articles and lead to unacceptable loss in the quality of the journals. Then, as budgets contract and OA publishing is promoted, journals may have to cease publication, and be permanently lost to the academic community and the public. These threats to scholarly publications are disturbing and should be addressed by the Journal. 
Economic realities and increasing user demands are moving us towards internet-only access. It becomes attractive to consider publication as an internet-only journal, with protection from public access for a period of 6-12 months, while available to subscribers or by payment of a fee to view each article. With this comes the promise of exploiting the interactive capabilities of the internet: to perform our own data analysis on study results, post research protocols, discuss case reports or have instant correspondence with authors. The Journal is moving towards even greater use of the internet. The future is not clear, but the opportunities are real.

\section{Vers des revues en libre accès}

\section{Expansion des archives électroniques du Journal canadien d'anesthésie}

Le projet d'expansion des archives électroniques du Journal canadien d'anesthésie a été annoncé en janvier 2006. ${ }^{1}$ Le projet a été développé sur une période de deux ans par Donald Miller, notre rédacteur en chef, à la suite de discussions menées avec HighWire Press au sujet de la politique d'accessibilité des archives adoptée par certaines publications biomédicales. Tous les articles publiés dans le Journal peuvent désormais être recherchés sur Internet par le nom des auteurs, des mots clés ou la date de publication (www.cja-jca. org). Une liste des références ainsi que des liens vers les textes intégraux et les résumés sont disponibles pour les articles publiés depuis le $\mathrm{l}^{\text {er }}$ janvier 2000. Cette base de données contient des documents PDF et des résumés de tous les articles publiés de 1966 à 1999. L'année suivant la publication, les articles ne sont accessibles qu'aux abonnés, ou sur une base de paiement à la carte pour les non-abonnés. Ensuite, ils deviennent disponibles gratuitement sur Internet.

\section{Une politique du Journal qui évolue}

Ce n'est pas la première fois qu'un changement majeur a lieu dans la politique de publication du Journal. Les "Proceedings of the Society", publiés pour la première fois en 1952, sont devenus le journal trimestriel "Journal de la société canadienne des anesthésistes » en 1954. Cette publication s'est transformée en "Journal canadien d'anesthésie » en 1987 et est désormais une publication mensuelle. Ces changements reflètent un lectorat à la hausse ainsi que la maturation des politiques de rédaction et d'affaires de la revue. Depuis 1997, la version imprimée du Journal est complétée par le CD-Rom de "The Electronic Anesthesia Library (TEAL) »; de plus, un format de texte intégral en ligne existe depuis sept ans. Le projet d'expansion des archives électroniques va de pair avec l'amélioration des services aux lecteurs et respecte le modèle traditionnel de fonctionnement des publications biomédicales. Ce projet se fonde sur le fait que les recettes des abonnements et de la publicité proviennent du matériel protégé publié durant les six à douze derniers mois. Nous ne prévoyons pas de manque à gagner, mais seulement des coûts modestes liés à la numérisation des fichiers PDF ainsi qu'aux frais actuels d'hébergement du site.

\section{L'émergence des périodiques électroniques}

La méthode de publication traditionnelle de journaux biomédicaux, disponibles uniquement en version imprimée, est mise en péril par l'émergence de la publication électronique et de la distribution en ligne. ${ }^{2}$ Les supports numériques offrent la possibilité d'une dissémination plus rapide de quantités illimitées d'informations; en outre, ils éliminent les coûts d'impression et de distribution. La publication en ligne est perçue comme une solution à la «crise des périodiques ", causée par l'augmentation rapide du nombre de publications biomédicales dans les années 90, les coûts de production plus élevés, les retards de publication, les prix en hausse des journaux pour les abonnés privés ainsi que par les restrictions budgétaires des institutions, lesquelles ont limité la capacité des bibliothèques à acheter des périodiques. Comme on pouvait s'y attendre, les sociétés plus modestes offrant des journaux imprimés sur une base d'abonnements ont eu peine à répondre aux coûts plus élevés et certaines ont cessé leurs parutions, dont "CRNA: Clinical Forum for Nurse Anesthetists». ${ }^{3}$ Avec l'accès mondial à une technologie Internet à bas prix, les modèles traditionnels de publication ne sont plus viables.

Les bases de données académiques ont été hébergées sur Internet afin d'offrir un archivage permanent ainsi qu'un accès facile aux articles épuisés ou publiés exclusivement sur support électronique. On peut obtenir un article particulier d'un auteur ou une revue complète sur des sites tels que PubMed Central, qui héberge plus de 200 périodiques, y compris BMJ, CMAJ, Canadian Family Physician, et PloS Medicine (www.pubmedcentral.nih.gov). Alors que davantage de revues rendent accessibles en ligne des articles et des suppléments, seulement quelques-unes sont désor- 
mais disponibles qu'en format imprimé. Entre 2003 et 2005 , la publication d'articles exclusivement en ligne a doublé de 7 à $14 \%$ (de 60/861 à 128/917 de tous les articles) dans 138 revues à fort impact (y compris le "New England Journal of Medicine ", "Pediatrics", "CMAJ» et le "British Journal of Anaesthesia»). ${ }^{4}$ La plupart des périodiques ont recours aux liens hypertextes pour renvoyer aux citations et envoyer des courriers électroniques d'information aux abonnés, mais peu profitent déjà pleinement du potentiel des fonctions interactives.

Pourtant, les habitudes de lecture des abonnés aux journaux ont évolué, d'une dépendance aux journaux imprimés et aux bibliothèques à l'utilisation de l'accès informatique à la littérature tirée de l'Internet.

\section{Politique de libre accès}

Le concept de publication en libre accès $(\mathrm{OA}-$ « Open Access ») a gagné de la vitesse ces cinq dernières années. ${ }^{5}$ Ce terme fait référence à la distribution et à la disponibilité sans restriction de la recherche scientifique et des publications via Internet. Des groupes intéressés cherchent à promouvoir le libre accès : le Budapest Open Access Initiative (2002-2003), le Bethesda Statement on Open Access Publishing (2003) et la Berlin Declaration on Open Access to Knowledge in the Science and Humanities (2003). Ces groupes sont partisans d'une politique de libre accès aux publications, laquelle va plus loin que le simple libre accès à tous les textes intégraux de PubMed Central et satisfait aux conditions suivantes : ${ }^{6}$

1) Le ou les auteur(s) et détenteur(s) des droits d'auteur accorde(nt) un droit d'accès libre, irrévocable, mondial et perpétuel, ainsi qu'un droit de copier, utiliser, distribuer, transmettre et exposer l'œuvre à des fins publiques, ainsi que le droit de créer et de distribuer des travaux dérivés en n'importe quel format numérique et ce, pour tout objectif responsable, sous réserve de l'attribution appropriée à l'auteur ou aux auteurs, et le droit d'imprimer en petit nombre des exemplaires pour leur utilisation personnelle.

2) Une version complète de l'œuvre et de tous les matériaux additionnels, comprenant une copie de la permission telle qu'énoncée ci-dessus, dans un format électronique standard adéquat, est immédiatement déposée lors de la première publication auprès d'au moins un dépositaire en ligne associé à une institution d'enseignement, une société savante, une agence gouvernementale ou toute autre organisation reconnue qui cherche à promouvoir le libre accès, la distribution sans restriction, l'interopérabilité et l'archivage à long terme (dans le domaine des sciences biomédicales, PubMed Central constitue un tel dépositaire).
La définition du libre accès est discutable, mais tout matériel d'érudition qui est en libre accès devrait être librement accessible sur Internet. Dans le monde de la publication en libre accès, connue sous le nom de " route d'or », les revues avec comité de lecture peuvent être archivées dans un dépositaire institutionnel qui rend leurs articles immédiatement disponibles en ligne. L'archivage du libre accès par les auteurs, ou « route verte", permet aux auteurs de rendre leurs articles librement accessibles après l'impression ou avant l'impression en tant que non révisés par les pairs, en plus de leur publication dans une revue. ${ }^{2}$

\section{Questions de droits d'auteur}

Dans la publication de matériel imprimé, l'attribution et l'usage adéquats d'un article publié sont régis par les lois de droits d'auteur ou copyright. Ces lois protègent la propriété intellectuelle des auteurs médicaux et visent à équilibrer les droits des auteurs, des éditeurs et des détenteurs des droits d'auteur avec le droit de la société en général d'avoir accès à la connaissance. Elles permettent une "utilisation équitable » pour copier, publier et distribuer l'œuvre en tout ou en partie sans la permission de l'auteur dans certaines circonstances. ${ }^{7}$ Au Canada, les auteurs d'œuvres originales, y compris de logiciels, ont automatiquement le droit de restreindre l'accès de tiers à l'utilisation, la publication, la lecture et la modification de leur ouvre. Il n'existe pas de clauses spéciales pour les archives électroniques, mais les lois sur les droits d'auteur sont plus difficiles à appliquer au support électronique. Dans le monde de la publication en libre accès, les auteurs peuvent conserver leur droit d'auteur pour les articles qu'ils ont archivés, mais les accords de licence peuvent outrepasser ces droits. Dans le cas du Journal, les articles archivés étaient et demeurent sous les clauses de droit d'auteur de la Société canadienne des anesthésiologistes (SCA). De fait, le rédacteur en chef, au nom de la SCA, a octroyé la permission à HighWire de donner suite au projet. Les mêmes clauses de droit d'auteur s'appliquent aux articles publiés dans le Journal avant 2000, en format PDF, ou plus tard, en format html.

\section{Impressions et points de vue sur la publication en libre accès}

L'accès universel et libre à la littérature scientifique est perçu comme un bien commun qui profite à l'humanité. Alors qu'une connaissance partagée devrait promouvoir la recherche et l'éducation dans les pays en voie de développement, les éditeurs soulignent le fait qu'ils fournissent déjà gratuitement au monde entier les contenus des revues par le biais de programmes internationaux. Les groupes de pres- 
sion de patients pensent qu'ils ont le droit de voir les résultats de recherches au sujet de maladies spécifiques qui sont subventionnées par des fonds publics. Les organismes subventionnaires ont réfléchi à l'octroi de bourses conditionnel au libre accès, mais la plupart des organismes de financement importants en Amérique du Nord et au Royaume-Uni (National Institutes of Health, le Conseil de recherches en sciences humaines, les Instituts de recherche en santé du Canada, Génome Canada et Wellcome), qui sont en faveur du libre accès, ne parviennent pas à couvrir les coûts de publication. Durant un temps, le NIH a rendu obligatoire le dépôt des articles publiés au PubMed Central; toutefois, en 2005, cette clause a été changée en une exigence volontaire suite à des plaintes des éditeurs. Le respect de cette exigence a d'abord été bas : seulement 2 à 3 $\%$ des articles admissibles ont été rendus disponibles sur PubMed Central. ${ }^{2}$

Une distribution mondiale opportune des articles devrait accroître la visibilité et le lectorat, mais l'impact du libre accès sur les taux de citation n'a pas encore été clairement déterminé. Un compte rendu au sujet d'articles publiés dans la même revue, en libre accès ou non, de juin à décembre 2004, relève que $14,2 \%$ des articles étaient en libre accès (les auteurs payant pour la publication) et que ceux-ci étaient cités deux fois plus souvent que les articles qui n'étaient pas en libre accès. ${ }^{8}$ Un sondage électronique incluant les auteurs ayant soumis des manuscrits au BMJ, Archives of Diseases in Childhood et au Journal of Medical Genetics en 2004, a montré que les auteurs n'étaient que peu familiers avec le libre accès et qu'ils ne considéraient pas ce critère dans leur choix d'un journal pour la soumission de leurs manuscrits. ${ }^{9}$ Les auteurs souhaitent voir leurs articles publiés dans une revue avec comité de pairs, avec libre accès ou non. Pour le moment, les universités ne s'entendent pas sur le fait de savoir si les publications en libre accès devraient ou non être utilisées dans les décisions de permanence et de promotion.

\section{Coûts de publication}

Les auteurs écrivent leurs manuscrits à l'aide d'un traitement de texte afin de produire des fichiers électroniques qu'ils envoient aux rédacteurs via Internet. Les rédacteurs distribuent les manuscrits à des évaluateurs qui renvoient leurs commentaires, à nouveau via Internet. Des modifications sont apportées au manuscrit par l'auteur, le rédacteur, l'évaluateur et le compositeur typographe pour produire le fichier électronique final, en général au format PDF. Jusque là, le coût de préparation du manuscrit final est le même pour les revues à publication traditionnelle imprimée ou électroniques/sur Internet. Toutefois, le coût de distribution des journaux imprimés est bien plus élevé que celui des journaux électroniques ou sur Internet, en raison des coûts supplémentaires occasionnés par l'impression et le port. Les économies générées par le passage d'une revue médicale canadienne enregistrée, le Clinical and Investigative Medicine, au format Internet exclusif, ont permis au journal de survivre et de continuer à publier.

Malgré les économies anticipées dans les coûts d'impression et de distribution, le passage d'un matériel imprimé à un matériel électronique n'élimine pas complètement les coûts de publication. ${ }^{10}$ Les défenseurs de la publication imprimée (ou opposants à la publication en ligne) soutiennent que la publication électronique ne réduit pas les coûts de publication. On a prétendu que la publication électronique pouvait être aussi coûteuse que la publication imprimée, le coût par article pour chaque médium ayant été estimé à \$ 3000 (\$ 1000-6000). ${ }^{11}$ Le prix d'achat des revues est connu, bien que des tarifs avantageux puissent être octroyés aux abonnements pour les institutions, les lots de journaux électroniques et les licences de site. ${ }^{12}$ Des analystes ont tenté de comparer les coûts de publication de périodiques dans plusieurs domaines, ou entre les secteurs à but lucratif et à but non lucratif, en calculant le coût moyen par article sur la base du prix et du nombre d'articles ou de citations énumérés dans la base de données Journal Citation Reports du International Standards Institute (ISI) (www.journalprices.com). Si l'on utilise les données ISI 2002 et les prix de 2006 (en dollars US), en ce qui concerne les revues médicales, le coût moyen par article ou par citation est trois fois plus élevé pour un journal à but lucratif que pour un journal à but non lucratif $(\$$ 3,1 vs 10,5 par article ou $\$ 2,9$ vs 8,5 par citation). L'avantage d'un calcul du prix par article ou citation permet d'explorer de nouveaux modèles commerciaux à appliquer à la publication en ligne. Toutefois, ce modèle révèle des différences de coûts qui sont difficilement explicables.

Les coûts de publication des revues scientifiques dépendaient traditionnellement du maintien d'une base d'abonnés. Des coûts de publication plus élevés ont été tenus pour responsables de l'escalade des prix des journaux. Néanmoins, les éditeurs de publications à but lucratif gardent confidentiels les budgets et marges de profit justifiant ces augmentations. Avec la publication en libre accès, le recouvrement des coûts dépend souvent d'un modèle d'entreprise "l'auteur paie (pour publier)» ou "l'utilisateur paie (pour visionner) ». La Public Library of Science charge des frais de publication aux auteurs ainsi qu'aux sponsors 
de recherche (annulés si l'auteur n'a pas de fonds disponibles) de 1250 à $2500 \$$ par article pour la publication dans leurs journaux PloS. Ce montant est calculé en fonction des frais liés au traitement et à la production du manuscrit, mais pas des coûts de commercialisation, d'hébergement en ligne et du personnel de la rédaction. ${ }^{11}$ BioMed Central offre aux institutions des abonnements annuels qui permettent de couvrir ces frais pour leurs auteurs, mais le prix fluctue en fonction du nombre d'articles hébergés l'année précédente. Les publications à but non lucratif, tels que les revues des sociétés, fonctionnent sur des budgets nettement plus bas en s'appuyant sur des évaluateurs pairs bénévoles et une administration du bureau de rédaction subventionnée. Les revenus de la publicité pour les journaux imprimés représentaient un profit qui finançait d'autres activités de la société. Ces sources de fonds n'ont pas encore été transférées aux publications en ligne. La profitabilité de l'entreprise pourrait avoir diminué à cause de cela, mais cela ne constitue pas un changement dans les coûts de production réels d'un journal.

Les rédacteurs ne peuvent que peu s'appuyer sur les coûts reportés de publication, lesquels varient considérablement, et doivent prendre des décisions qui répondent aux besoins de chaque publication. La plupart des revues scientifiques reconnues continuent à être imprimées en plus de posséder une version en ligne, renonçant ainsi à maximiser les réductions potentielles de coûts. De nouvelles publications exclusivement électroniques ont également proliféré ; ces dernières sont publiées par de petits groupes ou par un individu seul fonctionnant avec un budget limité. La publication de tels journaux a été possible grâce au libre accès à des systèmes de logiciels de gestion de journal, comme par exemple le Public Knowledge Project Open Journal Systems (OJS) (http://pkp.sfu. $\mathrm{ca} / ? \mathrm{q}=\mathrm{ojs}$ ), financé par le gouvernement fédéral, qui gère tous les aspects d'un article révisé par les pairs.

\section{Prévoir l'avenir}

En faisant l'analogie entre les publications scientifiques électroniques et un écosystème fragile de l'environnement, Forsman et Denison ${ }^{13}$ déclarent que : «Lorsque les ressources sont universellement disponibles et que la propriété est peu claire ou non protégée, l'exploitation arrive fréquemment.» Toutefois, la publication sur Internet peut répondre au besoin du public d'avoir accès à l'information tout en conservant les valeurs traditionnelles qui assurent la qualité d'un périodique. S'il y a une pression continue de la part des organismes subventionnaires et du gouvernement pour un libre accès, fournissant ainsi un accès public immédiat aux articles, les revues cliniques risqueront de perdre leurs revenus liés aux abonnements. Des réductions de coûts pourraient avoir pour résultat la publication d'articles non révisés et engendrer une perte inacceptable de la qualité des revues. Ensuite, alors que les budgets diminuent et que la publication en libre accès est encouragée, les revues pourraient devoir cesser de publier, et constituer une perte irrévocable pour la communauté et le public. Ces menaces qui planent sur les revues d'érudition sont troublantes et devraient être abordées par le Journal.

Les réalités économiques et les exigences accrues des utilisateurs nous poussent vers un accès par Internet exclusivement. Il est tentant de concevoir la publication comme journal uniquement en ligne, avec un accès restreint au public pendant 6 à 12 mois, moyennant un abonnement ou le paiement d'un montant pour la consultation de chaque article. La promesse d'exploitation des capacités interactives de l'Internet vient avec ce système : effectuer sa propre analyse des données sur des résultats d'étude, publier des protocoles de recherche, discuter des rapports de cas ou correspondre instantanément avec les auteurs. Le Journal se dirige vers un usage encore plus étendu de l'Internet. L'avenir est incertain, mais les opportunités sont réelles.

\section{References}

1 Miller DR, Members of the Canadian Journal of Anesthesia Editorial Board. Canadian Journal of Anesthesia: 2006 and beyond. Can J Anesth 2006; 53: $1-5$.

2 Albert KM. Open access: implications for scholarly publishing and medical libraries. J Med Libr Assoc 2006; 94: $253-62$.

3 Gunn IP. Death of a journal: lost opportunities, new challenges, or both? CRNA 2000; 11: 197-201.

4 Schriger DL, Ouk S, Altman DG. The use of the World Wide Web by medical journals in 2003 and 2005: an observational study. Pediatrics 2007; 119: 53-60.

5 Bluh P. "Open access," legal publishing, and online repositories. J Law Med Ethics 2006; 34: 126-30.

6 PMC Open access publishing. The Bethesda Statement on Open Access Publishing, April 2003. Available from URL; h tetp://www.pubmedcentral.gov/about/openaccess (accessed February 25, 2007).

7 Waters DJ. Managing digital assets in higher education: an overview of strategic issues. Association of Research Libraires (ARL). A bi-monthly report 2006; 244: 1-19. Available from URL; http://www.arl. org/resources/pubs/br/br244 (accessed February 25, 2007). 
8 Eysenbach $G$. Citation advantage of open access articles. PLoS Biology 2006; 4: el57.

9 Schroter S, Tite L. Open access publishing and authorpays business models: a survey of authors' knowledge and perceptions. J R Soc Med 2006; 99: 141-8.

10 Frank $M$. Access to the scientific literature - a difficult balance. N Engl J Med 2006; 354: 1552-5.

11 Frank $M$, Reich $M$, Ra'anan $A$. A not-for-profit publisher's perspective on open access. Serials review 2004; 30: 281-7. Available from URL; citeulike.org/user/ lschiff/article/72139 (accessed February 25, 2007).

12 Jeon-Slaughter H, Herkovic AC, Keller MA. Economics of scientific and biomedical journals: where do scholars stand in the debate of online journal pricing and site license ownership between libraries and publishers? First Monday March 2005; 10 (3). Available from URl; http://firstmonday.org/issues/issuel0_3/jeon/ index.htm (accessed February 25, 2007).

13 Forsman RB. Life and death on the coral reef: an ecological perspective on scholarly publishing in the health sciences. J Med Libr Assoc 2005; 93: 7-15. 